\title{
Copper-catalyzed cyanation of aryl halides with sodium nitroprusside in polyethylene glycol 400 (PEG-400)
}

\author{
Minhua Jiang, ${ }^{\text {a,b }}$ Tangjun Yuan, ${ }^{a}$ Fei Yi, ${ }^{\text {a }}$ and Junmin Chen*a \\ ${ }^{a}$ Department of Chemistry, Jiangxi Normal University, Nanchang 330022, P. R. China \\ ${ }^{b}$ School of New Energy Science \& Engineering, Xinyu University, Xinyu 338000, P. R. China \\ E-mail:jxnuchenjm@,163.com
}

DOI: $\underline{\text { http://dx.doi.org/10.3998/ark.5550190.p009.590 }}$

\begin{abstract}
A simple and efficient CuI/PEG-400 catalytic protocol for the cyanation of aryl halides employing sodium nitroprusside, $\mathrm{Na}_{2}\left[\mathrm{Fe}(\mathrm{CN})_{5} \mathrm{NO}\right] \cdot 2 \mathrm{H}_{2} \mathrm{O}$, as a new cyanation reagent was developed. The present cyanation reagent shows higher activity and gave the desired products in yields of up to $96 \%$. In the present protocol, a variety of aryl bromides and iodides were cyanated smoothly with a wide range of substrate scope.
\end{abstract}

Keywords: Cyanation, copper, aryl halides, cyanides, PEG-400

\section{Introduction}

Aryl carbonitriles constitute an important class of compounds that exist widely in natural products, pharmaceuticals, and agrochemicals. ${ }^{1}$ For example, the synthesis of fluvoxamine, an antidepressant which functions as a selective serotonin reuptake inhibitor, utilizes 4-(trifluoromethyl)benzonitrile as a key intermediate. ${ }^{2}$ Furthermore, the nitrile group serves as an intermediate for a multitude of transformations into other important functional groups such as benzoic acids/esters, amidines, amides, imidoesters, benzamidines, amines, heterocycles such as thiazoles, oxazolidones, triazoles and tetrazoles, aldehydes, etc. ${ }^{3}$ Traditional routes towards aryl nitriles include the Rosenmund-von Braun reaction from aryl halides or diazotization of anilines ${ }^{4}$ and a subsequent Sandmeyer reaction. ${ }^{5}$ A drawback to traditional methods is the use of stoichiometric amounts of copper(I) cyanide as a cyanating agent, which leads to considerable amounts of heavy metal waste. For these reasons, much attention has been given to the development of efficient and practical methods for the synthesis of aryl nitriles. Of these transformations, transition-metal-mediated cyanation of aryl halides represents one of the most convenient approaches. 
The first Pd-catalyzed cyanation of haloarenes was introduced in 1973 by Takagi et al. using aryl bromides and iodides with potassium cyanide as cyanating agent. ${ }^{6}$ Later, various kinds of $\mathrm{CN}$ source were explored, such as palladium-, nickel- and copper-catalyzed methods, using $\mathrm{KCN},{ }^{7,8} \mathrm{NaCN},{ }^{9,10} \mathrm{Me}_{3} \mathrm{SiCN},{ }^{11-13}{ }^{n} \mathrm{Bu}_{3} \mathrm{SnCN} \cdot \mathrm{Zn}(\mathrm{CN})_{2}{ }^{14-17}$ and $\mathrm{CuSCN}^{18}$ as cyanating agents. However, $\mathrm{KCN},{ }^{n} \mathrm{Bu}_{3} \mathrm{SnCN}$, and $\mathrm{NaCN}$ are extremely poisonous; $\mathrm{Me}_{3} \mathrm{SiCN}$ is sensitive to moisture and easily liberates hydrogen cyanide. These drawbacks seriously restrict their application. ${ }^{19}$ Recently, Beller and co-workers reported Pd-catalyzed cyanation of aryl halides by the use of $\mathrm{K}_{4} \mathrm{Fe}(\mathrm{CN})_{6}$, as a cyanide source, ${ }^{20}$ this is non-toxic and cheaper as compared to alkalimetal cyanides such as $\mathrm{KCN}$. Due to its significant advantages, $\mathrm{K}_{4}\left[\mathrm{Fe}(\mathrm{CN})_{6}\right]$ has been paid increasing attention as a cyanating agent in cyanation of aryl halides. ${ }^{21-27}$ Among various catalysts for the transition metal-catalyzed cyanation of aryl halides, palladium compounds have been widely investigated, while less expensive copper catalysts received little attention. The first case of copper-catalyzed cyanation of aryl halides was reported by Beller and co-workers. ${ }^{19}$ Subsequently, many methods of copper-catalyzed cyanation of aryl halides with $\mathrm{K}_{4} \mathrm{Fe}(\mathrm{CN})_{6}$ were developed. ${ }^{24-27}$ However, specific ligands and/or organic solvents were needed to obtain high yield. Very recently, Wang reported an efficient methodology for the cyanation of aryl iodides using copper salt as the catalyst, $\mathrm{K}_{4}\left[\mathrm{Fe}(\mathrm{CN})_{6}\right]$ as the cyanide source, and water as the solvent under microwave heating at $140{ }^{\circ} \mathrm{C} .{ }^{28}$ Subsequently, Leadbeater reported a similar methodology of cyanation of aryl iodides with $\mathrm{K}_{4}\left[\mathrm{Fe}(\mathrm{CN})_{6}\right]$ using water and tetraethylene glycol mixture as solvent. ${ }^{29}$ However, the copper-catalyzed cyanation of aryl- and heteroaryl bromides requires the use of a ten-fold amount of the rather expensive ligand $N, N^{\prime}$-dimethylethylenediamine relative to the copper salt. ${ }^{28,30}$ This feature is thus less attractive for large-scale applications. Recently, Cheng reported a safe and practical procedure for the copper-mediated cyanation of indoles by the combination of amine and ammonium as a safe cyanide source. However, the substrate was limited to various indoles. ${ }^{31}$ As a consequence, the development of a cheaper and environmentally friendly catalyst system for cyanation of aryl halides is greatly desirable. Research in our laboratory has been focused around the application of PEG-400 as a solvent for preparative chemistry, particularly transition-metal catalyzed coupling reactions. ${ }^{32-34}$ To the best of our knowledge, $\mathrm{Na}_{2}\left[\mathrm{Fe}(\mathrm{CN})_{5} \mathrm{NO}\right] \cdot 2 \mathrm{H}_{2} \mathrm{O}$ as the cyanide source in cyanation reactions has not so far been reported. Here, we describe for the first time its use as a cyanating agent for the general synthesis of benzonitriles with the objective of developing a clean, cheap, and efficient methodology.

\section{Results and Discussion}

In our initial study, cyanation of iodobenzene was chosen as a model reaction, and selected results from our screening experiments are summarized in Table 1. In the absence of base, only $8 \%$ yield was obtained using copper iodide (0.1equiv) as the catalyst, $\mathrm{Na}_{2}\left[\mathrm{Fe}(\mathrm{CN})_{5} \mathrm{NO}\right] \cdot 2 \mathrm{H}_{2} \mathrm{O}$ ( 0.3 equiv) as the cyanide source in PEG-400 under $\mathrm{N}_{2}$ at $120 \mathrm{C}$ for $20 \mathrm{~h}$ (Table 1, entry 1 ). The 
reaction afforded benzonitrile (2a) in $96 \%$ yield in the presence of $\mathrm{NaOH}$ (2.0 equiv) as base under the above reaction conditions, while only a moderate yield was obtained using $\mathrm{K}_{4}\left[\mathrm{Fe}(\mathrm{CN})_{6}\right]$ as cyanide source $(64 \%$, Table 1 , entry 2$)$. Then, various bases was screened, we found that weak base is ineffective under in the present of catalyst system, for example, only $13 \%$ and $21 \%$ yields were obtained in the case of $\mathrm{K}_{3} \mathrm{PO}_{4}$ and $\mathrm{K}_{2} \mathrm{CO}_{3}$ as base, respectively (Table 1 , entries 3, and 4). Reactions with other copper catalysts such as copper(II) oxide and copper(II) chloride proceeded with reduced yields (Table 1, entries 5, and 6). However, the reaction with copper(I) oxide gave the product in a moderate yield (Table 1, entry 7 ).

Table 1. Optimization of the reaction conditions

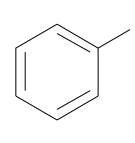

$1 \mathrm{a}$

$$
\text { I } \underset{[\mathrm{Cu}], \text { base, PEG-400 }}{\stackrel{\left.\mathrm{Na}_{2}(\mathrm{CN})_{5} \mathrm{NO}\right] \cdot 2 \mathrm{H}_{2} \mathrm{O}}{\longrightarrow}}
$$

\begin{tabular}{cccc}
\hline Entry $^{\mathrm{a}}$ & {$[\mathrm{Cu}]$} & base & Yield $^{\mathrm{b}}(\%)$ \\
\hline 1 & $\mathrm{CuI}$ & $/$ & 8 \\
\hline 2 & $\mathrm{CuI}$ & $\mathrm{NaOH}$ & $64^{\mathrm{c}}, 96$ \\
3 & $\mathrm{CuI}$ & $\mathrm{K}_{3} \mathrm{PO}_{4}$ & 13 \\
4 & $\mathrm{CuI}$ & $\mathrm{K}_{2} \mathrm{CO}_{3}$ & 21 \\
5 & $\mathrm{CuO}$ & $\mathrm{NaOH}$ & 30 \\
6 & $\mathrm{CuCl}_{2}$ & $\mathrm{NaOH}$ & 24 \\
7 & $\mathrm{Cu}_{2} \mathrm{O}$ & $\mathrm{NaOH}$ & 56 \\
$8^{\mathrm{d}}$ & $\mathrm{CuI}$ & $\mathrm{NaOH}$ & 93 \\
$9^{\mathrm{e}}$ & $\mathrm{CuI}$ & $\mathrm{NaOH}$ & 97 \\
\hline
\end{tabular}

${ }^{\mathrm{a}}$ Reaction conditions: iodobenzene $(1.0 \mathrm{mmol}), \mathrm{Na}_{2}\left[\mathrm{Fe}(\mathrm{CN})_{5} \mathrm{NO}\right] \cdot 2 \mathrm{H}_{2} \mathrm{O}(0.3 \mathrm{mmol}), \mathrm{Cu}$ source (0.1 mmol), $\mathrm{NaOH}$ (2.0 equiv), solvent PEG-400 (2 mL), at $120 \mathrm{C}$ for $20 \mathrm{~h}$ under $\mathrm{N}_{2}{ }^{\mathrm{b}}$ Isolated yield. ${ }^{\mathrm{c}}$ using $\mathrm{K}_{4}\left[\mathrm{Fe}(\mathrm{CN})_{6}\right]$ as a cyanation reagent. ${ }^{\mathrm{d}}$ Reaction temperature $100 \mathrm{C}$. ${ }^{\mathrm{e}}$ Reaction temperature at $140 \mathrm{C}$.

Subsequently, the reaction temperature was screened and it was found that the reaction at $100{ }^{\circ} \mathrm{C}$ can give the desired product in $93 \%$ yield (Table 1 , entry 8 ), while a yield of $97 \%$ was obtained at $140 \mathrm{C}$ (Table 1, entry 9). Therefore, by a systematic variation of the reaction parameters, the optimal catalytic system was determined, involving the use of $\mathrm{CuI}(10 \mathrm{~mol} \%)$, $\mathrm{Na}_{2}\left[\mathrm{Fe}(\mathrm{CN})_{5} \mathrm{NO}\right] \cdot 2 \mathrm{H}_{2} \mathrm{O}$ (0.3 equiv), and $\mathrm{NaOH}$ (2 equiv.) in PEG-400 at $120 \mathrm{C}$ for $20 \mathrm{~h}$.

With these encouraging results in hand we were interested in the scope and limitation of our new protocol. The cyanation of various aryl halides was first examined. As summarized in Table 2 , both electron-rich and electron-deficient aryl iodides were converted into the corresponding aryl cyanides smoothly in good to excellent yields (Table 1, entries 1-11). Cyanation of the 
sterically hindered $o$ - $\mathrm{MeOC}_{6} \mathrm{H}_{4} \mathrm{I}$ required a higher reaction temperature for satisfactory conversion and afforded the desired product in $75 \%$ yield (Table 3, entry 6). Interestingly, the cyanation reaction was able to tolerate a wide range of functional groups, for example, aryl iodides such as $p-\mathrm{FC}_{6} \mathrm{H}_{4} \mathrm{I}, p-\mathrm{ClC}_{6} \mathrm{H}_{4} \mathrm{I}$, and $p$ - $\mathrm{BrC}_{6} \mathrm{H}_{4} \mathrm{I}$ were converted into the desired nitriles at $100{ }^{\circ} \mathrm{C}$ in excellent yields ( $>89 \%$; Table 2 , entries $\left.7-9\right)$. Even a substrate containing an amino group was well tolerated and did not suffer $\mathrm{N}$-arylation (Table 2, entry 10).

Table 2. Cyanation of various aryl halides

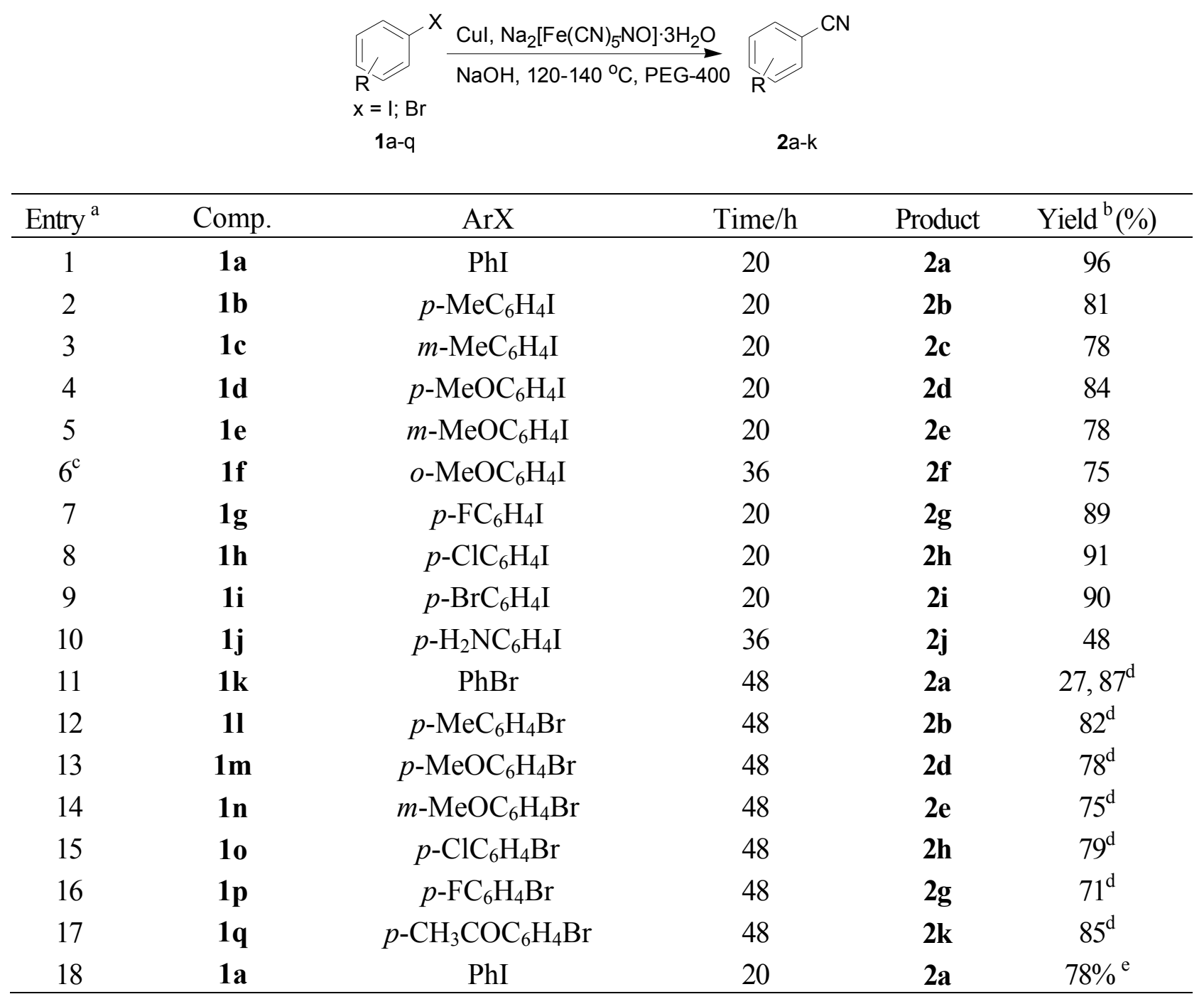

${ }^{a}$ Reaction conditions: phenyl halide $(1.0 \mathrm{mmol}), \mathrm{Na}_{2}\left[\mathrm{Fe}(\mathrm{CN})_{5} \mathrm{NO}\right] \cdot 2 \mathrm{H}_{2} \mathrm{O}(0.3 \mathrm{mmol}), \mathrm{CuI}(0.1$ mmol), $\mathrm{NaOH}$ (2.0 equiv), solvent PEG-400 (2 mL), at $120 \mathrm{C}$ for $20 \mathrm{~h}$ under $\mathrm{N}_{2}$.

${ }^{\mathrm{b}}$ Isolated yield.

${ }^{\mathrm{c}} \underline{\text { Reaction temperature } 140 \mathrm{C}}$.

${ }^{\mathrm{d}}$ L-proline $(0.2 \mathrm{mmol})$ was added.

e The reaction was performed on a $50 \mathrm{mmol}$ scale. 
Then we tested the activity of aryl bromides under our reaction conditions (Table 1, entries 11-17). However, a low yield was found under the iodide-optimized reaction conditions (27\%; Table 2, entry 11). Recently, Ma and co-workers reported that L-proline was a versatile privileged ligand and can accelerate copper-assisted Ullmann-type reactions. ${ }^{35}$ Stimulated by these results; we realized that the amino acids might be suitable ligands for the similar reactions. We were pleased to find that cyanation of bromobenzene also proceeded well in excellent yield by adding L-proline (0.2 equiv) as a ligand at $120 \mathrm{C}$ for $48 \mathrm{~h}(89 \%$, Table 2 , entry 1$)$. This method was successfully extended to aryl bromides deactivated by electron donating groups, such as methyl, methoxyl, (Table 2, entries 12-14) or electron-withdrawing groups such as chloro and fluoro (Table 2, entries 15, 16). Furthermore, 4-bromoacetophenone was also converted into the corresponding product in $85 \%$ yield (Table 2, entry 17). The protocol is also suitable for larger-scale reactions; for example, a 78\% yield of benzonitrile was obtained when the cyanation of iodobenzene was conducted on a $50 \mathrm{mmol} \mathrm{scale}$ (Table 2, entry 18). It is important to stress that aryl chlorides did not work under our protocol, which proved the inactivity of the $\mathrm{C}-\mathrm{Cl}$ bond in this catalysis.

\section{Conclusions}

In summary, we have developed a simple and efficient CuI/PEG-400 catalytic protocol for the cyanation of aryl halides employing $\mathrm{Na}_{2}\left[\mathrm{Fe}(\mathrm{CN})_{5} \mathrm{NO}\right] \cdot 2 \mathrm{H}_{2} \mathrm{O}$ as a new cyanation reagent. Compared with traditional $\mathrm{K}_{4}\left[\mathrm{Fe}(\mathrm{CN})_{6}\right]$, the nitroprusside reagent shows greater activity. Under the present protocol a variety of aryl iodides and bromides were cyanated smoothly with a wide range of substituent scope in good to excellent yields.

\section{Experimental Section}

General. All reagents were obtained from commercial sources and used without further purification. The reactions were carried out under a nitrogen atmosphere. All products were isolated by column chromatography on silica gel (200-300 mesh) using petroleum ether (b.p. 60$90{ }^{\circ} \mathrm{C}$ ) / ethyl acetate mixture as eluate. Compounds described in the literature were characterized. The ${ }^{1} \mathrm{H}$ and ${ }^{13} \mathrm{C}$ NMR spectra were recorded on a Bruker AC-400 (400 MHz) spectrometer with TMS as an internal standard. Melting points were determined on an XT-4 electrothermal micro-melting-point apparatus. Mass spectra were determined on a Finnigan 8230 mass spectrometer.

Procedure for the cyanation of aryl iodides. A $25 \mathrm{~mL}$ flask was charged with aryl iodide (1.0 $\mathrm{mmol}), \mathrm{Na}_{2}\left[\mathrm{Fe}(\mathrm{CN})_{5} \mathrm{NO}\right] \cdot 2 \mathrm{H}_{2} \mathrm{O}$ (89 mg; $\left.0.3 \mathrm{mmol}\right), \mathrm{NaOH}$ (80 mg; $\left.2.0 \mathrm{mmol}\right), \mathrm{CuI}$ (19 mg; 0.1 mmol), and PEG-400 (2 mL). The flask was evacuated and filled with nitrogen (three times) and 
heated to $120-140{ }^{\circ} \mathrm{C}$ for $20-48 \mathrm{~h}$, and then cooled to room temperature. The mixture was extracted with ethyl ether $(3 \times 10 \mathrm{~mL})$. The combined ethyl ether extracts were washed with brine, dried over $\mathrm{Na}_{2} \mathrm{SO}_{4}$, filtered and evaporated under reduced pressure, the residue was purified by flash column chromatography (ethyl acetate / petroleum ether mixture as eluate) to afford the desired products 2 (Table 2, entries 1-10).

Procedure for the cyanation of aryl bromides. A $25 \mathrm{~mL}$ flask was charged with aryl bromides (1.0 mmol), $\mathrm{Na}_{2}\left[\mathrm{Fe}(\mathrm{CN})_{5} \mathrm{NO}\right] \cdot 2 \mathrm{H}_{2} \mathrm{O}$ ( $\left.89 \mathrm{mg} ; 0.3 \mathrm{mmol}\right), \mathrm{NaOH}$ (80 mg; $\left.2.0 \mathrm{mmol}\right), \mathrm{CuI}$ (19 mg; $0.1 \mathrm{mmol})$, L-proline (23 mg, $0.2 \mathrm{mmol})$, and PEG-400 (2 mL). The flask was evacuated and filled with nitrogen (three times) and heated to $120-140{ }^{\circ} \mathrm{C}$ for $20-48 \mathrm{~h}$, and then cooled to room temperature. The mixture was extracted with ethyl ether $(3 \times 10 \mathrm{~mL})$. The combined ethyl ether extracts were washed with brine, dried over $\mathrm{Na}_{2} \mathrm{SO}_{4}$, filtered and evaporated under reduced pressure; the residue was purified by flash column chromatography (ethyl acetate / petroleum ether mixture as eluate) to afford the desired products 2 (Table 2 entries 11-17).

Benzonitrile (2a), ${ }^{8}$ Table 2, entries 1 and 11. Colorless oil (99 mg, 96\%). ${ }^{1} \mathrm{H}$ NMR (400 MHz, $\left.\mathrm{CDCl}_{3}\right): \delta$ 7.57-7.61 (m, 2H), 7.55-7.56 (m, 1H), 7.39-7.45 (m, 2H). ${ }^{13} \mathrm{C} \mathrm{NMR} \mathrm{(100} \mathrm{MHz,}$ $\left.\mathrm{CDCl}_{3}\right): \delta 158.0,145.2,123.7,115.6 . \mathrm{MS}(\mathrm{EI}, \mathrm{m} / z): 103\left[\mathrm{M}^{+}\right]$.

4-Methylbenzonitrile (2b). ${ }^{23}$ Table 2, entries 2 and 12. Colorless oil (95 mg, 81\%). ${ }^{1} \mathrm{H}$ NMR $\left(400 \mathrm{MHz}, \mathrm{CDCl}_{3}\right): \delta 7.54(\mathrm{~d}, J 6.8 \mathrm{~Hz}, 2 \mathrm{H}), 7.27(\mathrm{~d}, J 6.4 \mathrm{~Hz}, 2 \mathrm{H}), 2.43(\mathrm{~s}, 3 \mathrm{H}) .{ }^{13} \mathrm{C}$ NMR $(100$ $\mathrm{MHz}_{\mathrm{CDCl}}$ ): $\delta 143.7,132.0,129.9,119.1,109.3,21.8$. MS (EI, $\left.m / z\right): 117\left[\mathrm{M}^{+}\right]$.

3-Methylbenzonitrile (2c). ${ }^{8}$ Table 2, entry 3: Colorless oil (91 mg, 78\%). ${ }^{1} \mathrm{H}$ NMR (400 MHz, $\left.\mathrm{CDCl}_{3}\right): \delta 7.46(\mathrm{~s}, 2 \mathrm{H}), 7.416-7.32(\mathrm{~m}, 2 \mathrm{H}), 2.40(\mathrm{~s}, 3 \mathrm{H}) .{ }^{13} \mathrm{C} \mathrm{NMR}\left(100 \mathrm{MHz}, \mathrm{CDCl}_{3}\right): \delta 139.2$, 134.0, 132.3, 129.3, 188.9, 111.2, 20.5. MS (EI, $m / z): 117\left[\mathrm{M}^{+}\right]$.

4-Methoxybenzonitrile (2d). ${ }^{8}$ Table 2, entries 4 and 13. Colorless oil (112 mg, 84\%). ${ }^{1} \mathrm{H}$ NMR $\left(400 \mathrm{MHz}, \mathrm{CDCl}_{3}\right): \delta 7.56(\mathrm{~d}, J 8.0 \mathrm{~Hz}, 2 \mathrm{H}), 6.94(\mathrm{~d}, J 8.4 \mathrm{~Hz}, 2 \mathrm{H}), 3.83(\mathrm{~s}, 3 \mathrm{H}) .{ }^{13} \mathrm{C}$ NMR $\left(\mathrm{CDCl}_{3}, 100 \mathrm{MHz}\right) \delta 162.8,134.0,119.3,114.8,103.9,55.5 . \mathrm{MS}(\mathrm{EI}, \mathrm{m} / z): 133\left[\mathrm{M}^{+}\right]$.

3-Methoxybenzonitrile (2e). ${ }^{8}$ Table 2, entries 5 and 14. Colorless oil (104 mg, 78\%). ${ }^{1} \mathrm{H}$ NMR $\left(400 \mathrm{MHz}, \mathrm{CDCl}_{3}\right): \delta 7.41-7.32(\mathrm{~m}, 1 \mathrm{H}), 7.26-7.21(\mathrm{~m}, 1 \mathrm{H}), 7.16-7.11(\mathrm{~m}, 2 \mathrm{H}), 3.86(\mathrm{~s}, 3 \mathrm{H}) .{ }^{13} \mathrm{C}$ NMR (100 MHz, $\left.\mathrm{CDCl}_{3}\right): \delta 139.7,134.6,132.6,129.9,188.9,111.8,55.7 . \mathrm{MS}(\mathrm{EI}, \mathrm{m} / z): 133$ $\left[\mathrm{M}^{+}\right]$.

2-Methoxybenzonitrile (2f) ${ }^{\mathbf{8}}$ Table 2, entry 6. Colorless oil (100 mg, 75\%). ${ }^{1} \mathrm{H}$ NMR (400 $\mathrm{MHz}_{\mathrm{CDCl}}$ ): $\delta 7.55(\mathrm{~d}, J 7.2 \mathrm{~Hz}, 2 \mathrm{H}), 7.03-6.98(\mathrm{~m}, 2 \mathrm{H}), 3.93(\mathrm{~s}, 3 \mathrm{H}) .{ }^{13} \mathrm{C} \mathrm{NMR}(100 \mathrm{MHz}$, $\left.\mathrm{CDCl}_{3}\right): \delta 161.2,134.8,133.7,120.8,116.5,111.4,101.7,56.4$. MS (EI, $\left.m / z\right): 133\left[\mathrm{M}^{+}\right]$.

4-Fluorobenzonitrile (2g), ${ }^{8}$ Table 2, entries 7 and 16. White solid (108 mg, 89\%). Mp 34$36{ }^{\circ} \mathrm{C}$; ${ }^{1} \mathrm{H}$ NMR $\left(400 \mathrm{MHz}, \mathrm{CDCl}_{3}\right): \delta 7.42(\mathrm{dd}, J 8.8 \mathrm{~Hz}, 5.2 \mathrm{~Hz}, 2 \mathrm{H}), 7.05(\mathrm{t}, J 8.8 \mathrm{~Hz}, 2 \mathrm{H}) .{ }^{13} \mathrm{C}$ $\mathrm{NMR}\left(\mathrm{CDCl}_{3}, 100 \mathrm{MHz}\right) \delta 163.8,161.9,136.6,128.8,128.7,116.0,115.8 . \mathrm{MS}(\mathrm{EI}, \mathrm{m} / z): 121$ $\left[\mathrm{M}^{+}\right]$.

4-Chlorobenzonitrile (2h), ${ }^{8}$ Table 2 , entries 8 and 15. White solid (126 mg, 91\%). M. P. 90-93 ${ }^{\circ} \mathrm{C} .{ }^{1} \mathrm{H}$ NMR $\left(400 \mathrm{MHz}, \mathrm{CDCl}_{3}\right): \delta 7.53(\mathrm{~d}, J 8.4 \mathrm{~Hz}, 2 \mathrm{H}), 7.39(\mathrm{~d}, J 8.4 \mathrm{~Hz}, 2 \mathrm{H}) .{ }^{13} \mathrm{C}$ NMR $\left(\mathrm{CDCl}_{3}, 100 \mathrm{MHz}\right) \delta 139.5,133.4,129.7,118.0,110.8 . \mathrm{MS}(\mathrm{EI}, \mathrm{m} / z): 137\left[\mathrm{M}^{+}\right]$. 
4-Bromobenzonitrile (2i), ${ }^{23}$ Table 2, entry 9. White solid (164 mg, 90\%). M. P. 109-102 ${ }^{\circ} \mathrm{C} ;{ }^{1} \mathrm{H}$ NMR (400 MHz, $\left.\mathrm{CDCl}_{3}\right): \delta 7.64(\mathrm{~d}, J 8.4 \mathrm{~Hz}, 2 \mathrm{H}), 7.53(\mathrm{~d}, J 8.4 \mathrm{~Hz}, 2 \mathrm{H}) .{ }^{13} \mathrm{C} \mathrm{NMR}\left(\mathrm{CDCl}_{3}, 100\right.$ $\mathrm{MHz}) \delta 133.3,132.7,127.9,118.0,111.3$. MS (EI, $m / z): 181\left[\mathrm{M}^{+}\right]$.

4-Aminobenzonitrile (2j), ${ }^{\mathbf{2 8}}$ Table 2, entry 10. White solid $(57 \mathrm{mg}, 48 \%)$. M. P. 82-84 ${ }^{\circ} \mathrm{C} .{ }^{1} \mathrm{H}$ NMR (400 MHz, $\left.\mathrm{CDCl}_{3}\right): \delta 7.42(\mathrm{~d}, J 8.4 \mathrm{~Hz}, 2 \mathrm{H}), 6.65(\mathrm{~d}, J 8.4 \mathrm{~Hz}, 2 \mathrm{H}), 4.167(\mathrm{br}, 2 \mathrm{H}) .{ }^{13} \mathrm{C}$ $\mathrm{NMR}\left(\mathrm{CDCl}_{3}, 100 \mathrm{MHz}\right): \delta 150.5,133.8,120.1,114.4,100.0$. MS (EI, $\left.m / z\right): 118\left[\mathrm{M}^{+}\right]$.

4-Acetylbenzonitrile (2k), ${ }^{8}$ Table 2, entry 17. White solid (123 mg, 85\%). M. P. 76-79 ${ }^{\circ} \mathrm{C} .{ }^{1} \mathrm{H}$ NMR (400 MHz, $\left.\mathrm{CDCl}_{3}\right): \delta 8.04(\mathrm{~d}, J 8.4 \mathrm{HZ}, 2 \mathrm{H}) 7.77(\mathrm{~d}, J 8.4,2 \mathrm{H}), 2.65(\mathrm{~s}, 3 \mathrm{H}) .{ }^{13} \mathrm{C}$ NMR $\left(\mathrm{CDCl}_{3}, 100 \mathrm{MHz}\right): \delta 196.5,134.0,132.6,128.7,118.0,116.5,26.8$. MS (EI, $\left.m / z\right): 145\left[\mathrm{M}^{+}\right]$.

\section{Acknowledgements}

We are grateful for the financial support of Science and Technology Planning Project of Jiangxi Province, China (20142BAB203007).

\section{References}

1. Kleemann, A.; Engel, J.; Kutscher, B.; Reichert, D.; Pharmaceutical substances: syntheses, patents, applications, 4th ed., Georg Thieme, Stuttgart. 2001.

2. $\quad$ Rock, M. -H.; Merhold A.; (Bayer AG), US 6, 162,942. 2000.

3. Larock, R. C.; Comprehensive Organic Transformations. A Guide to Functional Group Preparations; VCH Publishers: New York. 1989.

4. Sandmeyer, T.; Ber. Dtsch. Chem. Ges. 1884, 17, 1633. http://dx.doi.org/10.1002/cber.18840170219

5. $\quad$ Rosenmund, K. W.; Struck, E.; Ber. Dtsch. Chem. Ges. 1919, 52, 1749. http://dx.doi.org/10.1002/cber.19190520840

6. Takagi, K.; Okamoto, T.; Sakakibara, Y.; Ohno, A.; Oka S.; Hayama, N. Bull. Chem. Soc. Jpn. 1975, 48, 3298.

http://dx.doi.org/10.1246/bcsj.48.3298

7. Beletskaya, I. P.; Sigeev, A. S.; Peregudov, A. S.; Petrovskii, P. V. J. Organomet. Chem. 2004, 689, 3810 . http://dx.doi.org/10.1016/j.jorganchem.2004.07.019

8. Sundermeier, M.; Zapf, A.; Beller, M. Angew. Chem. Int. Ed. 2003, 42, 1661. http://dx.doi.org/10.1002/anie.200250778

9. Zanon, J.; Klapars, A.; Buchwald, S. L. J. Am. Chem. Soc. 2003, 125, 2890. http://dx.doi.org/10.1021/ja0299708

10. Anderson, B. A.; Bell, E. C.; Ginah, F. O.; Harn, N. K.; Pagh, L. M.; Wepsiec, J. P. J. Org. Chem. 1998, 63, 8224. 
http://dx.doi.org/10.1021/jo9808674

11. Sundermeier, M.; Mutyala, S.; Zapf, A.; Spannenberg, A.; Beller, M. J. Organomet.Chem. 2003, 684, 50 .

http://dx.doi.org/10.1016/S0022-328X(03)00503-5

12. Chatani, N.; Hanafusa, T. J. Org. Chem. 1986, 51, 4714. http://dx.doi.org/10.1021/jo00374a041

13. Zieger, H. E.; Wo, S. J. Org. Chem. 1994, 59, 3838. http://dx.doi.org/10.1021/jo00093a016

14. Chobanian, H. R.; Fors, B. P.; Lin, L. S. Tetrahedron Lett. 2006, 47, 3303. http://dx.doi.org/10.1016/j.tetlet.2006.03.026

15. Jensen, R. S.; Gajare, A. S.; Toyota, K.; Yoshifujia, M.; Ozawab, F. Tetrahedron Lett. $\mathbf{2 0 0 5}, 46,8645$. http://dx.doi.org/10.1016/j.tetlet.2005.10.052

16. Hatsuda, M.; Seki, M. Tetrahedron 2005, 61, 9908. http://dx.doi.org/10.1016/j.tet.2005.06.061

17. Littke, A.; Soumeillant, M.; Kaltenbach, R. F.; Cherney, R. J.; Tarby, C. M.; Kiau, S. Org.Lett. 2007, 9, 1711. http://dx.doi.org/10.1021/o1070372d

18. Zhang, G.; Yu, J.; Hu, M.; Cheng, J. J. Org. Chem. 2013, 78, 2710. http://dx.doi.org/10.1021/jo3025829

19. Schareina, T.; Zapf, A.; Beller, M. Tetrahedron Lett. 2005, 46, 2585. http://dx.doi.org/10.1016/j.tetlet.2005.02.106

20. Schareina, T.; Zapf, A.; Beller, M. Chem. Commun. 2004, 1388. http://dx.doi.org/10.1039/B400562G

21. Yan, G.; Kuang, C.; Zhang, Y.; Wang, J. Org. Lett. 2010, 12, 1052. http://dx.doi.org/10.1021/ol1000439

22. Yeung, P. -Y.; So, C. -M.; Lau, C. -P.; Kwong, F. -Y. Angew. Chem. Int. Ed. 2010, 49, 8918. http://dx.doi.org/10.1002/anie.201005121

23. Chattopadhyay, K.; Dey, R.; Ranu, B. C. Tetrahedron Lett. 2009, 50, 3164. http://dx.doi.org/10.1016/j.tetlet.2009.01.027

24. Deblase, C.; Leadbeater, N. E. Tetrahedron 2010, 66, 1098. http://dx.doi.org/10.1016/j.tet.2009.11.016

25. Wang, J.; Ma, J.; Tian, X.; Yin, W. Adv. Synth. Catal. 2012, 354, 2301. http://dx.doi.org/10.1002/adsc.201200235

26. Tian, X.; Sun, Y.; Dong, C.; Zhang, K.; Liang, T.; Zhang, Y.; Hou, C. Chem. Lett. 2012, 41,719 . http://dx.doi.org/10.1246/c1.2012.719

27. Wen, Q.; Jin, J.; Mei, Y.; Lu P.; Wang, Y. Eur. J. Org. Chem. 2013, 4032. http://dx.doi.org/10.1002/ejoc.201300052 
28. Ren, Y.; Wang, W.; Zhao, S. Tian, X.; Wang, J.; Yin, W.; Cheng, L. Tetrahedron Lett. 2009, 50, 4595 .

http://dx.doi.org/10.1016/j.tetlet.2009.05.073

29. Blase, C. D.; Leadbeater, N. E. Tetrahedron 2010, 66, 1098.

http://dx.doi.org/10.1016/j.tet.2009.11.016

30. Zanon, J.; Klapars, A.; Buchwald, S. L.; J. Am. Chem. Soc. 2003, 125, 2890. http://dx.doi.org/10.1021/ja0299708

31. Liu, B.; Wang, J.; Zhang, B.; Sun, Y.; Wang, L.; Chen, J.; Cheng, J. Chem. Commun. 2014, $50,2315$.

http://dx.doi.org/10.1039/c3cc49339c

32. Chen, J.; Yuan, T.; Hao, W.; Cai, M. Tetrahedron Lett. 2011, 52, 3710.

http://dx.doi.org/10.1016/j.tetlet.2011.02.096

33. Chen, J.; Yuan, T.; Hao, W.; Cai, M. Catal. Commun. 2011, 12, 1463.

http://dx.doi.org/10.1016/j.catcom.2011.06.002

34. Chen, J.; Zhang, Y.; Hao, W. Zhang, R.; Yi, F. Tetrahedron 2013, 69, 613. http://dx.doi.org/10.1016/j.tet.2012.11.014

35. Ma, D.; Cai, Q. Acc. Chem. Res. 2008, 41, 1450.

http://dx.doi.org/10.1021/ar8000298 